\title{
THE EVOLUTIONARY CAUSES OF IRREGULARITY: EVIDENCE FOR AN IRREGULARIZATION BIAS IN MORPHOLOGICAL LEARNING
}

\author{
TIMOTHY J. O'DONNELL ${ }^{* 1}$ and KENNY SMITH ${ }^{2}$ \\ *Corresponding Author: timothy.odonnell@ mcgill.ca \\ ${ }^{1}$ Department of Linguistics, McGill University, Québec \\ ${ }^{2}$ Centre for Language Evolution, University of Edinburgh, UK
}

While inflectional morphology is broadly rule-governed, many inflectional paradigms admit some exceptions (e.g., the past tense of "go" is not "goed" but "went"). Regularity in form-meaning mapping permits generalization and facilitates learning, and regularity has been shown to emerge through language transmission (e.g. Kirby et al., 2008, 2015).

The presence of irregulars is more puzzling. One possibility is that irregulars are simply a by-product of processes which destroy regularity (e.g., minimisation of effort in production), which survive language transmission due to their high frequency (Kirby, 2001; Lieberman et al., 2007). Here we show that irregularity in the right place in a morphological system actually facilitates the learning of the productive parts of the system; therefore, irregularity might be favoured during language transmission, rather than simply being a by-product of other processes.

We build on a recent computational model (O'Donnell, 2015) which shows that high-frequency irregulars facilitate the learning of productive regular rules. This model treats learning as an inference problem, where learners infer the productivity of morphological processes, balancing a tradeoff between computation and storage. High-frequency items tend to be stored as wholes, rather than handled compositionally; productive computation is signaled by morphological processes which apply across a large number of low-frequency forms. Since there is pressure to store high-frequency regular forms, they detract from the productivity of regular inflectional processes. When high-frequency forms are instead irregular, regular rules generalize more easily.

We provide experimental evidence for this irregularization bias. 46 adult participants learned 48 novel inflected words, organized into six disjoint paradigms of eight words each (see Table 1). In two Fully Regular sets, all stems were inflected with a single regular suffix and occurred with equal frequency during training. The remaining four sets had eight stems that occurred with non-uniform frequency. In each set, seven stems occurred with a regular suffix while a single item took an irregular suffix. These sets differed in whether the the most frequent 
Table 1. Example word sets from the critical conditions, giving frequency of exposure in training for each word form and an example definition. Irregulars are highlighted in bold/red. The Fully Regular sets (not shown) have 8 stems, each occurring with frequency 12 and inflecting with a single regular suffix.

\begin{tabular}{cc}
\multicolumn{2}{c}{ Frequent Irregular Set } \\
\hline Frequency & Word \\
\hline 48 & shrunefench \\
17 & chunwobane \\
10 & yadnossane \\
6 & raldane \\
6 & shrumane \\
4 & benthane \\
3 & flovenane \\
3 & heespane \\
\hline Example definition: "shrunefench, \\
the tool used to shrunef" \\
\hline
\end{tabular}

\begin{tabular}{cc}
\multicolumn{2}{c}{ Frequent Regular Set } \\
\hline Frequency & Word \\
\hline 48 & fibimort \\
17 & dwilnebort \\
10 & spolakort \\
6 & moyport \\
6 & chervort \\
4 & glocktonort \\
3 & quideport \\
3 & dagzomuth \\
\hline Example definition: "fibimort, the \\
place where they make fibim" \\
\hline
\end{tabular}

item was irregular (Frequent Irregular sets) or regular (Frequent Regular sets). Participants were trained and tested over two days. Each day involved a training phase (auditory exposure to inflected forms plus definitions), followed by a test (participants were presented with an inflected word and asked if they had encountered it during training); these tests included trials which tested generalization of affixes, and trials which tested memorization of specific inflected forms.

Following the predictions of O'Donnell (2015), participants were more likely to generalize the regular affix from the Frequent Irregular set than the regular affix from the Frequent Regular set. This was not merely due to the higher frequency of regular suffixes from the Frequent Regular sets, since Fully Regular suffixes patterned with the Frequent Irregular suffixes (i.e. were highly productive, despite having frequency similar to the regular suffixes from the Frequent Regular sets). We also observed a storage advantage for irregulars: high-frequency irregulars were more likely to be accepted than frequency-matched high-frequency regulars.

These results demonstrate that high-frequency irregulars facilitate the generalization of regular rules for human language learners, or conversely that highfrequency regulars inhibit the generalization of a regular rule. This shows that systems in which high-frequency items are irregular have learnability advantages. In current work we are investigating whether this results in such systems emerging through language transmission; if so, irregularity might be explained by the same mechanisms already invoked to explain the evolution of regularity.

\section{Acknowledgements}

This project has received funding from the European Research Council (ERC) under the European Unions Horizon 2020 research and innovation programme (grant agreement 681942). 


\section{References}

Kirby, S. (2001). Spontaneous evolution of linguistic structure: an iterated learning model of the emergence of regularity and irregularity. IEEE Transactions on Evolutionary Computation, 5(2), 102-110.

Kirby, S., Cornish, H., \& Smith, K. (2008). Cumulative cultural evolution in the laboratory: an experimental approach to the origins of structure in human language. Proceedings of the National Academy of Sciences, USA, 105, 10681-10686.

Kirby, S., Tamariz, M., Cornish, H., \& Smith, K. (2015). Compression and communication in the cultural evolution of linguistic structure. Cognition, 141, 87-102.

Lieberman, E., Michel, J.-B., Jackson, J., Tang, T., \& Nowak, M. A. (2007). Quantifying the evolutionary dynamics of language. Nature, 449, 713-716.

O’Donnell, T. J. (2015). Productivity and reuse in language: A theory of linguistic computation and storage. Cambridge, Massachusetts and London, England: The MIT Press. 